\title{
Epstein-Barr Virus Infektionen
}

\section{Neue Aspekte zur Pathogenese und Klinik*}

\author{
E. Wilmes, $H$. Wolf \\ Klinik und Poliklinik für Hals-, Nasen- und Ohrenkranke, Ludwig-Maximilians-Universität München, Klinikum Großhadern \\ Direktor: Prof. Dr. med. E. Kastenbauer) \\ Max-von-Pettenkofer-Institut (Direktor: Prof. Dr. F. Deinhardt) der LMU München
}

\section{Zusammenfassung}

Das Epstein-Barr Virus (EBV) gehört zu den meist verbreiteten humanen Viren. Es ruft eine Reihe unterschiedlicher Krankheitsbilder, wie die akute infektiöse Mononukleose (IM), die chronisch aktive EBVInfektion (cEBV), das X-linked lymphoproliferative Syndrom (XLP), poly- und oligoklonale Lymphome bei Immunstörungen sowie das afrikanische Burkitt-Lymphom und das Nasopharynxkarzinom hervor. In dieser Arbeit wird auf die Pathogenese, Klinik und Diagnostik dieser Krankheitsbilder eingegangen. Dazu werden eigene Untersuchungen zur Latenz und Vermehrung von Epstein-Barr Virus vorgestellt. - Epstein-Barr Viren persistieren in den Ohrspeicheldrüsen gesunder Individuen und werden über den Speichel in die Mundhöhle ausgeschieden. Durch direkten Kontakt können sie übertragen werden und eine akute infektiöse Mononukleose hervorrufen. Erstmals gelang unserer Arbeitsgruppe der Nachweis der Virusvermehrung in der Tonsilla palatina in der akuten Phase der infektiösen Mononukleose.

\section{Epstein-Barr Virus Infections}

The Epstein-Barr virus (EBV) is among the most widespread of human viruses. It causes several different diseases, such as acute infectious mononucleosis (IM), chronic active EBV-infection ( $\mathrm{CEBV}$ ), the $\mathrm{x}$ linked lymphoproliferative syndrome (XLP), polyclonal and oligoclonal lymphomae in connection with immunologic disorders, as well as African Burkitt's lymphoma and nasopharyngeal carcinoma. Pathogenesis, clinical features and diagnosis are discussed. In this connection, special tests on the latency and multiplication of the Epstein-Barr virus are presented. - The Epstein-Barr virus persists in the parotid gland of healthy individuals and is secreted via saliva. It is transmitted by direct contact and responsible for infectious mononucleosis. For the first time we succeeded in proving the multiplication of the virus in the palatine tonsil during acute phase of infectious mononucleosis.
Das Epstein-Barr Virus (EBV) gehört zu den meist verbreiteten humanen Viren. Über $90 \%$ der erwachsenen Bevölkerung ist infiziert. Serologische Studien (Henle und Henle, 1976) haben gezeigt, daß Infektionen im Alter von weniger als 3 Jahren in Bevölkerungsgruppen mit niederem sozialen Milieu vorkommen, während diese in den Industrieländern erst im Adoleszentenalter („Studentenfieber") oder später erfolgt. Solche seroepidemiologischen Studien zeigten weiter, daß primäre Infektionen mit EBV in früher Kindheit entweder ohne Symptome (stille Feiung) oder sehr mild verlaufen. Im Adoleszentenalter hingegen führt die primäre Infektion häufiger zum klassischen Krankheitsbild der infektiösen Mononukleose (IM).

Es konnte weiterhin gezeigt werden, daß jeder, der Antikörper gegen EBV hat, über Lymphozyten verfügt (ca. 1 in 10000 ), welche EBV-Erbinformation in Form ringförmiger DNA enthalten. Diese Lymphozyten wachsen in der Gewebekultur unbegrenzt, wenn die sie kontrollierenden T-Zellen entfernt werden. Gereinigtes Virus kann in wenigen Tagen B-Lymphozyten von EBV-freien Personen in unbegrenzt wachsende Zellen umwandeln (immortalisieren).

Die Erstinfektion mit EBV führt zur infektiösen Mononukleose und ist Voraussetzung für die Entwicklung des Nasopharynxkarzinoms und des in Afrika endernischen Burkitt-Lymphoms. Zusätzlich verursacht das Epstein-Barr-Virus unter bestimmten Bedingungen weitere Krankheitsbilder, auf deren Klinik und Pathogenese im folgenden eingegangen werden soll.

\section{Akute infektiöse Mononukleose} (Normaler Ablauf einer EBV-Infektion)

Die infektiöse Mononukleose (IM) wird durch direkten Kontakt mit Speichel und Rachensekreten übertragen (,kissing disease“). Die Eintrittspforte ist die Mundhöhle. Nicht geklärt ist bisher, ob Lymphozyten (von denen bekannt ist, daß sie Rezeptoren für EBV besitzen) oder spezielle Epithelzellen - wie bei Varicella-zosterInfektionen - das Virus zunächst aufnehmen. Die exzessive Schwellung der Gaumentonsillen sowie des gesamten Waldeyerschen Rachenrings (WRR) wurde bisher als reaktive Proliferation von B-Lymphozyten (EBV besitzt mitogene Eigenschaften) gedeutet. 
Histologisch finden sich transformierte B-Lymphozyten in Tonsillen und in der Milz in Form von blasenartigen Ansammlungen. Sie ähneln B-Immunoblasten mit basophilem Zytoplasma und runden nukleolenhaltigen Zellkernen. Dieses Bild kann irrtümlich zur Diagnose eines malignen Lymphoms führen (Krüger, 1985). Gleichzeitig kommt es zur Reaktion des T-Zellsystems nach Art einer bunten Pulpahypertrophie.

Eigene Untersuchungen mit Hilfe der $\mathrm{Nu}$ kleinsäurehybridisierung zeigen, daß in der akuten Phase der infektiösen Mononukleose die Epstein-Barr-Viren in den Gaumentonsillen nicht nur vorhanden sind, sondern dort auch in epithelialen Zellen vermehrt werden (Abb. 1, 2).

\section{Methodik \\ Nukleinsäurehybridisierung}

Die Nukleinsäurehybridisierung basiert auf der thermodynamisch begünstigten Bildung von Doppelsträngen.

\section{In-situ-Hybridisierung (nach Wolf und Mitarb., 1984)}

Die DNA von Dünnschnitten (Gefrierschnitten oder Schnitten von fixiertem Gewebe) wird durch Hitzebehandlung in einzelsträngige Form überführt. Im Überschuß wird dann markierte einzelsträngige virusspezifische DNA $\left(10^{5} \mathrm{cpm}{ }^{3} \mathrm{H}\right.$-markierte EBV-DNA) zugegeben. Die markierte virale DNS wird in Hybriddoppelstränge gebunden (Inkubationsdauer 24 Stunden, $45^{\circ}, 0,6 \mathrm{M}$ $\mathrm{NaCl}, 50 \%$ Formamid) und kann nach Waschen (in $50 \%$ Formamid, $10 \mathrm{mM}$ TRIS, pH 7,5, $1 \mathrm{mM}$ EDTA, $600 \mathrm{mM} \mathrm{NaCl}$ über Nacht bei Raumtemperatur) durch Autoradiographie oder entsprechender enzymatischer Reaktion nachgewiesen werden.

\section{Southern-blot-Hybridisierung}

$10 \mathrm{~g}$ DNA aus den Geweben oder entsprechend geringere Mengen von DNA aus EBV-infizierten Zellen zur Kontrolle werden mit Restriktion : : zzym (Bam H I) verdaut, auf einem horizontalen 1\%igen Agaroses: elektrophoretisch getrennt und nach Denaturieren mit $0,5 \mathrm{~N} \mathrm{NaOH}$ und Neutralisation durch Kapillarwirkung auf Nitrozellulosemembran transferiert. Nach Absättigen verbleibender Bindungsstellen durch Präinkubationspuffer (Denhard-Lsg., $6 \times$ SSC-Lsg. und Hefe-RNA) wird durch Zugabe von frischem Hybridisierungspuffer mit $50 \%$ Formamid und $32 \mathrm{P}$-markierter EBV-DNA 16 Stunden bei $50^{\circ} \mathrm{C}$ inkubiert. Nach Waschen der Filter mit $1 \times$ SSC und $0,1 \%$ SDS bei Raumtemperatur und abschließendem Waschen mit $0,1 \times$ SSC, $0,1 \%$ SDS bei $65^{\circ} \mathrm{C}$ wird durch Autoradiographie mit Kodak Xomat-S bei $70^{\circ} \mathrm{C} 3$ bis 4 Tage entwickelt.

Wir nehmen an, daß die initiale Virusvermehrung nicht nur in den Tonsillen, sondern in allen lymphoepithelialen Organen des Waldeyerschen Rachenrings (WRR) stattfindet. Sie führt zur Zerstörung von Lymphozyten, entsprechend dem klinischen Bild einer nekrotisierenden Tonsillitis. Gleichzeitig mit der Virusreplikation im WRR kommt es zur hämatogenen Ausbreitung der Viren, die jetzt auch in peripheren B-Lymphozyten nachweisbar sind. Die Expression viraler Antigene führt zur Bildung von T-Lymphozyten, die für B-Lymphozyten zytotoxisch sind.

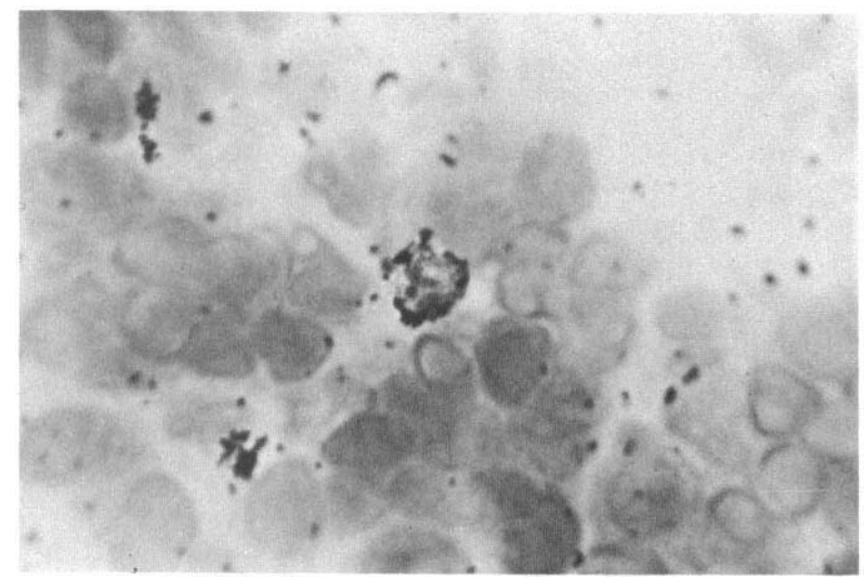

Abb. 1 In-situ-Hybridisierung einer Gaumentonsille bei IM. Die Granula entsprechen viraler (EBV) DNA.

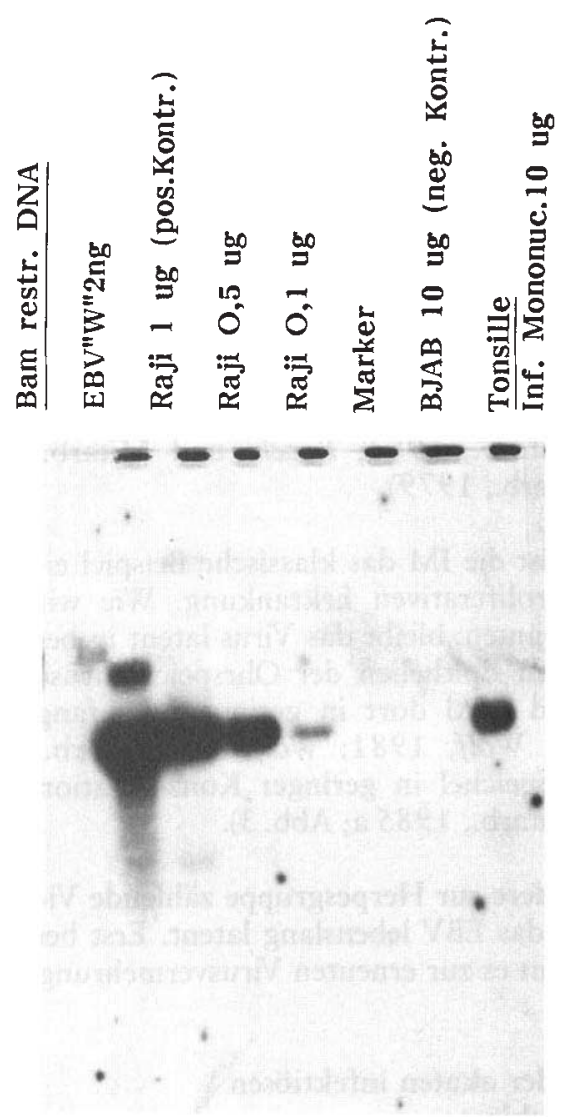

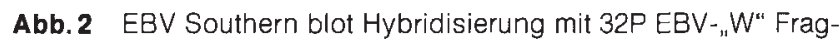
ment, 16 h $50^{\circ} \mathrm{C}, 50 \%$ Formamid, exponiert 67h. Nachweis viraler DNA.

Entsprechend sind bis zu $75 \%$ der lymphoiden Zellen im peripheren Blut T-Lymphozyten („,monozytoide“ Lymphozyten/retikuläre Reizformen).

Die Reaktion der T- mit den B-Zellen führt zum Vollbild des Pfeifferschen Drüsenfiebers. Es finden sich generalisierte, aber vorwiegend zervikale Lymphknotenschwellungen, was verständlich wird, da die Eintrittspforte für die Infektion im Oropharynx liegt. 


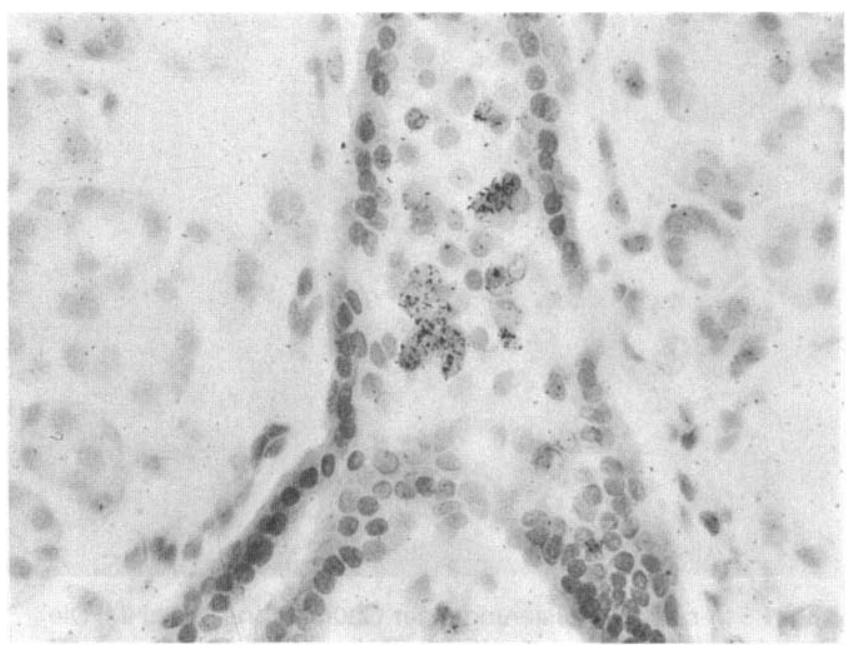

Abb.3 in-Situ-Hybridisierung einer Ohrspeicheldrüse. EBV-Genom-tragende Zellen.

Die EBV-infizierten B-Zellen werden zu Beginn der Infektion nur unvollständig kontrolliert bzw. nicht vor Vollendung des lytischen Vermehrungszyklus eliminiert. Dadurch sind diese in der Lage, eine Reihe viraler Proteine zu produzieren (EBNA, EA, VCA), die die Bildung von Antikörpern (Anti-EBNA, Anti-EA, Anti-VCA) anregen. Zusammen mit den zytotoxischen T-Zellen werden EBV-infizierte Zellen eliminiert (Svedmyr und Jondal, 1975; Meuer und Mitarb., 1983; Rocchi und Mitarb., 1977; Tosato und Mitarb., 1979).

Damit ist die IM das klassische Beispiel einer selbstlimitierten proliferativen Erkrankung. Wie wir schon früher zeigen konnten, bleibt das Virus latent in bestimmten differenzierten Epithelien der Ohrspeicheldrüse gesunder Personen und wird dort in geringem Umfang produziert (Wilmes u. Wolf, 1981; Wolf und Mitarb., 1984) und mit dem Speichel in geringer Konzentration freigesetzt (Yao und Mitarb., 1985 a; Abb. 3).

Wie andere zur Herpesgruppe zählende Viren (HSV, VZV) bleibt das EBV lebenslang latent. Erst bei Immunstörungen kommt es zur erneuten Virusvermehrung (siehe unten).

\section{Klinik der akuten infektiösen Mononukleose}

Die typischen Symptome der IM sind in Tab. 1 zusammengefaßt.

Tab. 1 Klinik der intektiösen Mononukleose

Uncharakteristisches Prodromalstadium

Lidödeme

Palatinale Petechien

Angina

Vorwiegend zervikale Lymphknotenschwellungen

Splenomegalie $(50 \%)$

Hepatomegalie (30\%)
Häufig als pathognomonisch angesehen wird ein kleinfleckiges Exanthem, das aber in $80 \%$ der Fälle Ampicillin-bedingt ist. Besonders die Tonsillen, aber auch der gesamte Waldeyersche Rachenring, zeigen das ganze Spektrum zwischen katarrhalischer Rötung bis zur ulzerierenden Entzündung. Typisch ist die Trias: Lymphadenitis, Fieber, Leukozytose (10000-20000 Zellen, $10 \%$ atypische mononukleäre Zellen).

Komplikationen, wie Milzrupturen kommen vor, sind aber selten. Trotzdem sollte die veränderte und vulnerable Struktur der Milz während der akuten und postakuten Phase Anlaß zur Vermeidung schwerer körperlicher Belastungen, z. B. durch Sport, sein. Normalerweise dauern die Symptome 1 bis 2 Wochen an. In einigen Fällen - meist Patienten, die dem Teenageralter entwachsen sind - verläuft die Infektion mit schweren subjektiven Krankheitszeichen, so daß eine stationäre Aufnahme erforderlich werden kann.

\section{Labordiagnostik}

Heterophile Antikörper, die Erythrozyten von verschiedenen Tieren agglutinieren (Paul Bunnel), sind im akuten Stadium nachweisbar. Die Ursache für das Auftreten solcher Antikörper ist wahrscheinlich durch die polyklonale Stimulation des Immunsystems durch EBV erklärbar, die durch die Bindung an den CR2-Rezeptor initiiert wird.

EBV-spezifische Seroreaktionen werden heute üblicherweise durchgeführt. IgM-Antikörper gegen VCA und IgG-Antikörper gegen EA (p54 oder die diffuse Komponente, EA-D) sind beweisend für das Vorliegen einer infektiösen Mononukleose. Ein einzelnes Serum genügt. Deutlich verbesserte und empfindlichere Testsysteme, die auf der Verwendung gentechnologisch hergestellter Antigene beruhen, sind kurz vor der Einführung (Wolf und Mitarb., 1987).

\section{Therapie der IM}

Eine kausale Behandlung ist nicht bekannt. Andererseits soll eine Tonsillektomie subjektive Beschwerden lindern und das Krankheitsbild abkürzen können ( $A$. Herrmann, 1963). Diese Meinung wird verschiedentlich von Kinderärzten und Virologen kontrovers diskutiert.

Nach unseren neuesten Vorstellungen, nach denen eine primäre Virusvermehrung im Waldeyerschen Rachenring stattfindet, ergibt sich durchaus ein rationaler Ansatzpunkt für die Tonsillektomie. Ausgehend von der klinischen Beobachtung einer Gesundung nach Tonsillektomie erklären wir diesen Effekt folgendermaßen:

Durch die Entfernung eines erheblichen Anteils virusproduzierender Zellen ist das Immunsystem bzw. sind zelluläre und humorale Abwehrmechanismen in der Lage rascher die Infektion zu beantworten und damit die Krankheitsdauer zu verkürzen bzw. die Schwere der Erkrankung zu mildern. Möglicherweise wird die immunologische Abwehr bei solchen schwerwiegenden Verläufen nicht ausreichend stimuliert. Sicher sind die meisten akuten IM ohnehin nicht operationsbedürftig, da sie in aller Regel auch ohne operativen Eingriff ausheilen. Allenfalls in den Fällen einer verlängerten Krankheitsdauer mit exzessiver Hyperplasie der Gaumenmandeln und erheblicher Belastung des Organismus erscheint eine Tonsillektomie indiziert. Unsere Ergebnisse unterstützen dieses Konzept. 
Darüber hinaus können klinische Studien so interpretiert werden, daß die Tonsillektomie zwar gravierende Krankheitssymptome mildert, aber die Immunlage im Hinblick auf eine erneute Erkrankung ungünstig beeinflussen kann (Sauerbrei und Mitarb., 1983).

\section{Die chronisch aktive EBV-Infektion (cEBV)}

Dieses erst kürzlich in der Literatur (Tobi und Mitarb., 1982; Hamblin und Mitarb., 1983; Jones und Mitarb., 1985; Strauss und Mitarb., 1985) bekannt gewordene Krankheitsbild ist durch schwer faßbare Symptome gekennzeichnet (Tab. 2).

Tab. 2 Symptome der chronisch aktiven EBV-Erkrankung

\begin{tabular}{|c|c|}
\hline Variabel: & $\begin{array}{l}\text { allgemeines Krankheitsgefühl } \\
\text { ausgeprägte Müdigkeit } \\
\text { Gedächtnisschwäche } \\
\text { Konzentrationsschwierigkeiten }\end{array}$ \\
\hline Häufig: & $\begin{array}{l}\text { Fieber } \\
\text { Entzündungen im Halsbereich } \\
\text { Frösteln }\end{array}$ \\
\hline
\end{tabular}

\section{Diagnostik}

Die EBV-spezifischen Immunfluoreszenzteste erlauben keine eindeutigen Aussagen. Die weitaus spezifischeren ELISA-Teste mit spezifischen Antigenen zeigen jedoch, daß Antikörper gegen EBNA-1 und -2 (Henle und Mitarb., 1987) und Membranantigen gp 350/250 (Jilg und Wolf, 1985) signifikant reduziert sind, während Antikörper gegen andere virale Proteine mehr oder weniger IM-Patienten oder gesunden Personen gleichen.

\section{Therapie}

Azyklovir und Interleukin 2 werden empfohlen. Der Nachweis freier viraler DNA (d.h. in nicht episomaler Form) in Lymphknoten-Biopsien oder peripheren Blut-Lymphozyten soll eine Azyklovir-Behandlung rechtfertigen, da dieses Medikament eine exzessive lytische Vermehrung beeinflussen soll (Raab-Traub und Flynn, 1986).

\section{Duncan-Syndrom (XLP)}

Diese sehr seltene Erkrankung wurde von Purtilo (1976) nach der Familie benannt, bei der sie erstmals beobachtet wurde. Die männlichen Abkömmlinge dieser Familie starben im frühen Kindesalter an der Infektion durch EBV. Bestimmte, noch unbekannte genetische Faktoren des X-Chromosoms (X-linked proliferative disease) bewirken, daß diese Infektion einen tödlichen Verlauf nimmt. Es ist noch ungeklärt, ob es sich hierbei entweder um ein malignes proliferatives Geschehen wie beim Burkitt-Lymphom oder um ein exzessiv reaktives Erkrankungsbild auf persistierende virusproduzierende Zellen im Blutkreislauf handelt.

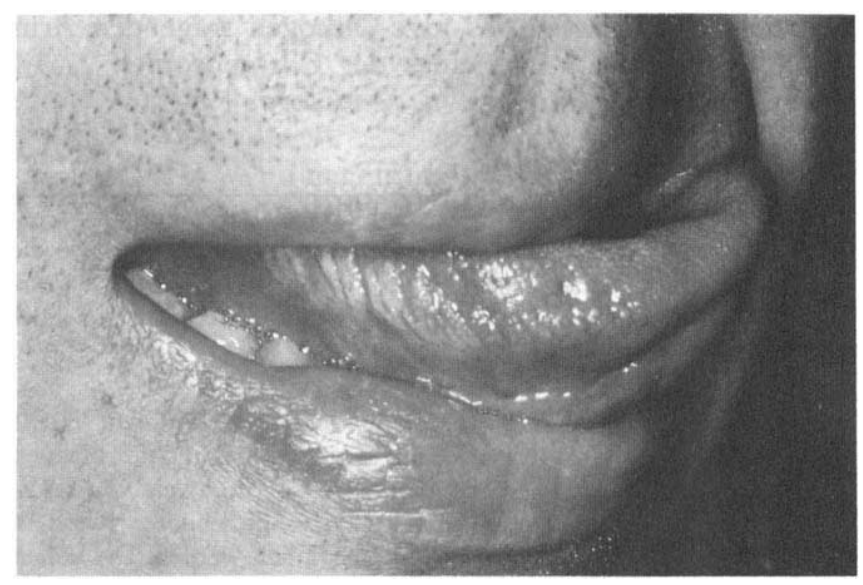

Abb. 4 Haarleukoplakie bei HIV-Infektion.

Immundefizienz und EBV-Infektion
Haarleukoplakie bei HIV-infizierten
Personen

Greenspan und Mitarb. (1985) beschrieben, daß Leukoplakien der Zunge bei AIDS-Patienten durch EBV-produzierende epitheliale Zellen charakterisiert sind. Sie führen dies zurück auf eine „Reaktivierung“ von latent EBV-enthaltenden epithelialen Zellen. In der Tat können diese Läsionen (Abb.4) als Analogie zu den Petechien in Begleitung der Primärinfektion (IM) und damit als charakteristisch für immunologisch nicht reaktive Wirtsorgane angesehen werden.

\section{Polyklonale B-Lymphome}

Als Folge einer geschwächten Immunitätslage (z. B. HIV-Infektionen) kommt es hier erneut zu einer Proliferation von B-Zellen mit EBV-Genomen und damit zur Bildung poly- oder oligoklonaler Lymphome (proliferativer Effekt von EBV). So wurde EBV-DNA in Lymphomen von Patienten nach Herz-, Nieren- oder Knochenmarktransplantationen nachgewiesen (Bieber und Mitarb., 1983; Hanto und Mitarb., 1982). Ähnliche Lymphome wurden häufig bei HIV-Infizierten beobachtet (Ziegler und Mitarb., 1982; Holzer u. Wolf, unveröffentlicht). Eine fehlende Immunsurveillance scheint das Wachstum multipler Zellklone zu begünstigen. Nach Knochenmarktransplantationen können diese Lymphome - die aus dem transplantierten Mark stammen - zum Tode innerhalb von 2 bis 3 Monaten führen (Schubach und Mitarb., 1982; Martin und Mitarb., 1984).

Bei Behebung der Immunsuppression bilden sich diese Lymphome oft zurück.

\section{Monoklonale Lymphome}

In seltenen Fällen kommt es durch Veränderung der infizierten Zellen oder durch Infektion unreifer B-Zellen zu Störungen der Zelldifferenzierung und als Folge zur Vermehrung von Zellen mit veränderten Zelloberflächen und damit zur Ausbildung monoklonaler Lymphome (siehe unten). Diese zeichnen sich durch Translokatio- 
nen aus, die stets den das myc-Onkogen tragenden Arm des Chromosoms 8 und eines der Chromosomen 2, 14 oder 22 betreffen (Manolov u. Manolova, 1972).

\section{Andere Erkrankungen}

Eine ganze Reihe weiterer Erkrankungen, wie die chronische lymphatische Leukämie oder der Morbus Hodgkin, wurden mit EBV in Zusammenhang gebracht, da diese erhöhte Antikörper gegen EBV-assoziierte Antigene zeigen.

Sorgfältige seroepidemiologische Studien (Henle u. Henle, 1979) zeigten aber auch das Gegenteil, nämlich das Fehlen von EBV-Antikörpern. Dies und der fehlende Nachweis von EBVDNA in Biopsien lassen einen direkten Zusammenhang zwischen EBV und diesen Erkrankungen unwahrscheinlich werden. Die hohen Antikörper sind wahrscheinlich Ausdruck eines Immundefektes der latent-infizierten B-Lymphozyten, der es erlaubt einer T-Zell-induzierten Elimination zu entkommen. Dadurch werden EBV-Antigene produziert, die wiederum eine humorale Antwort (Antikörper) hervorrufen.

In seltenen Fällen, in denen ein Morbus Hodgkin oder eine chronische lymphatische Leukämie einer schweren infektiösen Mononukleose folgten (Jehn und Mitarb. 1981), könnte die Erklärung in einer Wachstumsstimulation von vorhandenen prämalignen Zellen zu suchen sein.

\section{Maligne Tumoren}

Serologische Untersuchungen haben eine regelmäßige Erhöhung von Antikörpern gegen EBV-Antigene sowohl bei Patienten mit Burkitt-Lymphomen in Afrika (Henle u. Henle, 1967) als auch mit Nasopharynxkarzinomen (Henle und Mitarb., 1971; Henle u. Henle, 1979 a; Wilmes u. Wolf, 1981) gezeigt. Da das Virus jedoch weit verbreitet ist - über $90 \%$ der erwachsenen Bevölkerung haben Antikörper -, kann aufgrund der Serologie allein keine kausale Beziehung zwischen EBV und den beiden Neoplasien aufgestellt werden. Erst der direkte Nachweis von viraler DNA und viralen Antigenen in Tumormaterialien (Wolf und Mitarb., 1973; Wolf und Mitarb., 1975), in Zellsuspensionen (Desgranges und Mitarb., 1975 ) und in xenotransplantierten Tumoren in thymusaplastischen Mäusen (Klein und Mitarb., 1974 b) macht eine ursächliche Rolle von EBV wahrscheinlich. Unterstützt wird diese Behauptung durch Untersuchungen an Krallenaffen, die zeigen, daß Epstein-Barr-Virus letale lymphoproliferative Erkrankungen auslösen kann, in denen wiederum virale DNA in den Tumormaterialien gefunden werden. Darüber hinaus erscheint die Assoziation von EBV mit solchen Lymphomen auch wegen der in vitro immortalisierenden Eigenschaften naheliegend.

\section{Burkitt-Lymphom (BL)}

Burkitt-Lymphome treten nicht nur - wie zumeist angenommen - im Kieferwinkel, sondern in allen übrigen Regionen des Körpers auf. Befallen sind zumeist Kinder im Alter von 4 bis 10 Jahren. Für Virologen war das BL das Modell für einen virusbedingten Tumor.
Fialkow und Mitarb. (1976) zeigten, daß es sich beim Burkitt-Lymphom un einen monoklonalen, d. h. von einer einzigen Zelle ausgehenden 'Tumor handelt. Diese Klonalität ist ein Hinweis dafür, daß die Entwicklung eines Tumors im Organismus ein sehr seltenes Geschehen sein muß, möglicherweise aufgrund einer sehr strengen Immunüberwachung (Manolov u. Manolova, 1972).

Lenoir und Mitarb. (1982) sowie Taub und Mitarb. (1982) beschrieben die regelmäßige Translokation, betreffend das Chromosom 8 (das das myc-Gen trägt) sowie der Chromosomen 2, 14 oder 22. Die letzteren Chromosome kodieren verschiedene Immunglobulinketten. Diese Translokation findet sich auch bei allen anderen (vorwiegend nicht-afrikanischen) EBV-negativen BurkittLymphomen! Da durch die Translokation kontinuierlich verfügbares myc-Genprodukt die Ruhephase von Zellen aufhebt, wird die Differenzierung der Zellen möglicherweise verhindert. Als Konsequenz sind HLA-Antigene in veränderter Form exprimiert (Modrow und Mitarb., 1987) und virale Membranantigene ebenfalls in der Expression reduziert (Modrow und Wolf, 1986). Diese Zellen sind von einem intakten Immunsystem nicht mehr erkennbar.

$3 \%$ der afrikanischen $B \mathbf{L}$ und $75 \%$ der entsprechenden Erkrankungen außerhalb Afrikas enthalten keine EBV-DNA. Damit ist die Beziehung von EBV zu diesen Lymphomen keineswegs lückenlos. Das Virus ist möglicherweise nur ein Faktor in der Genese dieser Neoplasie, der durch einen oder mehrere andere ersetzt werden kann.

Andererseits muß EBV als ein wesentlicher Faktor für die Entstehung des afrikanischen BurkittLymphoms (inbesondere in Endemie-Gebieten wie Uganda) angesehen werden. So zeigten prospektive Studien hohe Antikörpertiter gegen EBV bei solchen Personen, die später ein Burkitt-Lymphom entwickelten (de The und Mitarb., 1987).

\section{Diagnose}

Die Serodiagnose für das BL ist bisher weniger ausführlich als für das NPC untersucht.

\section{Therapie}

Die Chemotherapie ist die Behandlung der Wahl. Obwohl die Ansprechrate "gut" ist, ist die Prognose ungünstig. Die 5-Jahres-Überlebensrate liegt bei ca. $25 \%$.

\section{Nasopharynxkarzinom (NPC)}

Die Infektion mit EBV ist ein essentieller Faktor in der Genese des NPC, wenngleich wie beim BL mit Sicherheit eine Virusinfektion allein nicht zur Bildung führt. Andererseits ist in jedem NPC virale EBV-DNA enthalten (s. o.). Während das NPC bei uns nur 3-5\% aller Neoplasien im Kopf-Hals-Bereich bzw. 0,3\% der Krebserkrankungen pro Jahr pro 100000 Einwohner ausmacht, stellt diese Erkrankung mit bis zu 40 pro 100000 in bestimmten Gegenden Südchinas (Guangxi) den häufigsten Tumor dar. 
Diese unterschiedliche Verteilung bzw. Erkrankungshäufigkeit war Anlaß, sowohl genetische als auch Umweltfaktoren zu untersuchen.

\section{Einfluß von genetischen Faktoren}

Bisherige genetische Untersuchungen erlauben keine eindeutige Aussage. Auf der einen Seite belegen ausführliche Studien von Simons und Mitarb. $(1975,1978)$ und Chan und Mitarb. (1981) eine signifikante Korrelation mit bestimmten HLA-Typen (AZ, BW46, B 17/BW58). Detaillierte Studien, vor allem in Familien mit mehreren Tumorfällen, werden allerdings notwendig sein, um genetische Einflüsse auf die Tumorentstehung zu erklären. Das Absinken der Tumorinzidenz in der zweiten Generation von chinesischen Auswanderern nach USA, vor allem wenn diese in ländlichen Gegenden leben, spricht eher gegen entscheidende genetische Einflüsse.

\section{Einflüsse von Umweltfaktoren}

Wegen der fraglichen und wohl allenfalls relativen Risikobeeinflussung genetischer Faktoren konzentrieren sich viele Untersuchungen zunehmend auf die Rolle von Umweltfaktoren. Für Nitrosamine, eine häufig genannte Quelle ist gesalzener Fisch, als Ursache für ein erhöhtes Risiko gibt es wenig Anhaltspunkte. Eine andere Quelle von möglichen Umwelteinflüssen wird dagegen in letzter Zeit systematisch erforscht. Es konnte gezeigt werden, daß eine Reihe von Heilpflanzen, die als Volksarznei in Form von Tees verabreicht werden, direkte Wirkungen auf das Epstein-Barr Virus ausüben (Zeng und Mitarb., 1983). Extrakte der erwähnten Pflanzen können die in Lymphozyten latenten Genome aktivieren und zumindest eine teilweise Reaktivierung von Epstein-Barr Virus bewirken. Diese Aktivierung des Virus führt letztlich zum Absterben der entsprechenden Wirtszellen. Es kann jedoch beobachtet werden, daß in der Gewebekultur solche „aktivierten Lymphozyten“ aufgrund einer virusbedingten Veränderung mit anderen Zellen ganz oder teilweise verschmelzen können, wenn sie in einem engen Kontakt zu solchen Zellen stehen (Bayliss u. Wolf, 1980, 1981; Wolf und Mitarb., 1981; Wilmes und Mitarb., 1983; Wolf und Mitarb., 1983). Dieser Vorgang könnte auch zu einer Übertragung viraler DNS in solche Zellen führen (hier epitheloide Zellen), die nicht direkt infizierbar sind. Obwohl hypothetisch, könnte dieser Mechanismus erklären, warum diese Tumorart vorwiegend auf den Nasenrachenraum beschränkt ist: Der Kontakt zwischen Lymphozyten und Epithelzellen (Lymphoepithel) ist im Waldeyerschen Rachenring sehr eng. Dies könnte auch die geographisch uneinheitliche Risikoverteilung erklären, wenn sich herausstellen sollte, daß die entsprechenden „Heilmittel“ nur in Gebieten mit hohem Risiko verwendet werden.

\section{Zur klinischen Relevanz der Virusassoziation des Nasopharynxkarzinoms}

Die einfachste diagnostische Methode ist die Untersuchung von Antikörpern gegen virale Antigene des Epstein-Barr Virus (Beispiel: Lymphknotenmetastase am Hals bei unbekanntem Primärtumor). Die Verbesserung der verwendeten Immunfluoreszenzteste durch Antikörperklassen-spezifische Untersuchungen haben diese Methode wegen ihrer Aussagekraft zu einem wichtigen Bestandteil der Patientenfürsorge gemacht (Wilmes und Mitarb., 1983). Da Immunfluoreszenzteste aber stets Antikörper- gegen Antigengemische testen und auch oft nicht einfach abzulesen sind, wurden neue Einzelantigen-spezifische, objektivierbare Tests entwickelt. Wolf und Mitarb. haben als Ansatz hierfür die Immunpräzipitation gewählt. Hierbei werden Lysate von virusproduzierenden Zellen, die alle viralen Proteine radioaktiv markiert enthalten, mit Seren inkubiert; die Immunkomplexe werden abgetrennt und nach elektrophoretischer Auftrennung durch Autoradiographie charakterisiert.

Es zeigt sich, daß Antikörper gegen die Proteine mit dem Molekulargewicht 138 kD regelmäßig und in einer hohen Konzentration nur bei Tumorpatienten vorhanden sind. Sie können daher als Test für diese Erkrankung dienen. Mit Hilfe der Gentechnologie gelingt es, dieses Protein in Bakterien ohne die störenden anderen viralen Proteine herzustellen. Diese Produkte ermöglichen nach entsprechender Reinigung die Entwicklung und Anwendung hochspezifischer Enzymimmunteste (Motz und Mitarb., 1986; Wolf und Mitarb., 1987).

\section{Verhütung von NPC durch Immunisierung (ein Ausblick)}

Die strikte Korrelation von NPC und EBV legt den Gedanken nahe, daß Verhütung, Verzögerung oder Modifikation der Infektion durch EBV die Entwicklung von NPC verhindern könnten. Als Kandidat für eine EBV-Vakzine wurde durch Schutzversuche in Krallenaffen das Membranantigen von EBV (gp 350/250) identifiziert (Epstein und Mitarb., 1985). Mit gentechnologischen Methoden ist es gelungen, dieses Antigen ohne Viruspräparation durch rekombinante, eukaryotische Zellen in Kultur zu erhalten. Damit steht ein wichtiges Ausgangsprodukt für eine EBV-Vakzine zur Verfügung.

Dieses Glykoprotein könnte auch für Risikopatienten mit Immunmangel oder auch für Erwachsene, die in der Kindheit nicht durch EBV infiziert wurden und im Falle einer Infektion schwerere Krankheitssymptome zu erwarten haben, einen Schutz gegen Erkrankung geben, ohne notwendigerweise eine EBV-Infektion lebenslang vollständig zu verhindern.

Um langfristigen Schutz und ausgeprägte zelluläre Immunantworten zu erhalten, wird der Einsatz rekombinanter Aktivimpfstoffe erwogen. Ein Beispiel ist die Einfügung zusätzlicher Information von z. B. EBV gp $350 / 250$ Membranantigen in das Genom von geeigneten Vakzinia-Impfstämmen. Da solche Impfungen wegen der reduzierten Antwort bei Mehrfachimpfungen nicht beliebig oft wiederholt werden können, wird für Gebiete mit bestimmten Risikokombinationen die Herstellung von Mehrfachrekombinanten betrieben. Motz und Mitarb. konnten sowohl das Oberflächenantigen von Hepatitis B als auch das EBV-Membranantigen gp 350/250 in ein Vakziniagenom einführen (Motz und Mitarb., 1986, 1987). In Gebieten wie Südchina, wo ca. $20 \%$ der Tumorerkrankungen primäre Leberkarzinome (assoziiert mit Hepatitis-B-Infektionen) und $23 \%$ NPC darstellen, eröffnen solche Impfstoffe interessante Perspektiven. 


\section{Literatur}

Bayliss, G. J., H. Wolf: An Epstein-Barr virus early protein induced cell fusion. Proc. Natl. Acad. Sci. USA 78 (1981) 7162

Bayliss, G. J., H. Wolf: Epstein-Barr virus induced cell fusion. Nature $287(1980)$

Bieber, C. P., R. L. Herberling, S. W. Jamieson: Lymphoma in cardiac transplant recipients: association with the use of cylosporin $\mathrm{A}$. prednisone and antihymocyte globulin. In: Immne Deficiency and Cancer: Epstein-Barr Virus and Lymphoproliferative Malignancies. Purtilo (ed.) Plenum Press, New York (1984) 309

Chan, S. H., N. E. Day, J. Wabrendorf: HLA antigens and nasopharyngeal carcinoma. In: IARC Annual Report. Lyon, 53 (1981)

Desgranges, $C$., $H$. Wolf, $G$. de Thé, K. Shanmugaratnam, N. Cammoun, R. Ellouz, G. Klein, N. Munoz, H. Hausen: Nasopharyngeal carcinoma. X. Presence of Epstein-Barr genomes in separated epithelial cells of tumours in patients from Singapore, Tunisia and Kenya. Int. J. Cancer $16(1975) 7$

De Thé, G., A. Geser, N.E. Day, P. M. Tukei, E. H. Williams, D. P. Beri, P. G. Smith, A. G. Dean, G. W. Bornkamm, P. Feorino, W. Henle Epidemiological evidence for causal relationship between EpsteinBarr virus and Burkitt's lymphoma from Ugandan prospective study. Nature 274 (1978 a) 756

Estein, M. A., A. J. Morgan, S. Finerty, B. J. Randle, J. K. Kirkwood: Protection of cottontop tamarins against Epstein-Barr virus-induced malignant lymphoma by a prototype vaccine. Nature 318 (1985) 287

Fialkow, P. J.: Clonal origin of human tumors. Biochem. Biophys. Acta $458(1976) 283$

Greenspan, J. S., D. Greenspan, E. T. Lennette, D. I. Abrams, M. A. Conant, V. Petersen, U. K. Freese: Replication of Epstein-Barr virus within the epithelial cells of oral "hairy " leukoplakia, an AIDS-associated lesion. New Engl. J. Med. 313 (1985) 1564

Hamblin, T., T. J. Hamblin, J. Jussain, A. N. Akbar, Y. C. Tang, J. L. Smith, D. B. Jones: Immunological reason for chronic ill health af ter infectious mononucleosis. In: Brit. Med. J., Vol. 287 (1983) 85

Hanto, D. W., G. Frizzera, K. J. Gajl-Peczalska et al.: Epstein-Barr virusinduced B-cell lymphoma after renal transplantation. Acylovir threrapy and transition from polyclonal to monoclonal B-cell proliferation. N. Engl. J. Med. 306 (1982) 913

Henle, G., W. Henle, G. Klein: Demonstration of two distinct components in the early antigen complex of Epstein-Barr virus infected cells. Int. J. Cancer 8 (1971) 272

Henle, G., W. Henle: Immunofluorescence, interference, and complement fixation technics in the detection of the herpes-type virus in Burkitt tumor cell lines. Cancer Res. 27 (1967) 2442

Henle, W. G. Henle, I. Anderson, I. Erngerb, G. Klein, C. A. Horwitz, G. Marklund, L. Rymo, C. Wellinder, S. E. Straus: Antibody responses to Epstein-Barr virus-determined nuclear antigen (EBNA-1) 1 and EBNA-2 in acute and chronic Epstein-Barr virus infection. Proc. Natl. Sci. USA 84 (1987) 570

Henle, W., G. Henle: Seroepidemiology of the virus. In: The Epstein-Barr Virus (M. A. Epstein and B. G. Achong, Eds.), Springer Verlag, Berlin, Heidelberg, New York (1979) 61

Herrmann, A.: die Mandelausschälung als Therapie der Monocytenangina. HNO 11 (1963) 219

Jehn, U., H. J. Sauer, H. Wolf, W. Wilmanns: Hodgkin's disease following infectious mononucleosis - a case report. Europ. J. Cancer 17 (1981) 447

Jilg, W., $H$. Wolf: Diagnostic significance of antibodies to the EpsteinBarr virus-specific membrane antigen gp 250 . J. Infect. Diseases $152(1985) 222$

Jilg, W., $H$. Wolf: The significance of antibodies against the Epstein-Barr virus-specific membrane antigen gp 240 in acute and latent EBV infections. In: Epstein-Barr Virus and Associated Diseases. Levine, $P$. H., D. V. Ablashi, G. R. Pearson, S. D. Kottardis (eds). Martinus Nijhoff Publishing, Boston (1985) 73

Jones, J. F., C. G. Ray, L. L. Minnich, M. J. Hicks, R. Kibler, D. O. Lucas. Evidence for active Epstein-Barr virus infection in patients with persistent, unexplained illness: elevated antiearly antigen antibodies. In: Ann. of Int. Med., Vol. 102, No. 1 (1985) 1

Klein, G., B. C. Giovanella, T. Lindahl, P. J. Fialkow, S. Singh, J. S. Stehlin: Direct evidence for the presence of Epstein-Barr virus DNA and nuclear antigen in malignant epithelial cells from patients with poorly differentiated carcinoma of the nasopharynx. Proc. Natl. Acad. Sci. USA 71 (1974 b) 4737

Krüger, G. R. F.: Klinische Immunpathologie. Kohlhammer Verlag (1985)
Lenoir, G. M., G. W. Bornkamm: Burkitt's lymphoma, a human cancer model for the study of the multistep development of cancer: Proposal for a new scenario. Advances in Viral Oncology $6(1987)$ in press

Manolov, G., Y. Manolova: Marker band in one chromosome 14 from Burkitt lymphomas. Nature, Lond., 237 (1972) 33

Martin, P. J., H. M. Shulman, W. H. Schubach et al.: Fatal Epstein-Barr virus-associated proliferation of donor B-cells after treatment of acute graft-versus-host-disease with a murine anti-T-cell antibody. Ann. Intern. Med. 101 (1984) 310

Meuer, S. C., I. C. Hodgdon, D. A. Cooper et al.: Human cytotoxic T-cell clones directed at autologous virus-transformed targets: further evidence for linage of genetic restriction to $\mathrm{T} 4$ and $\mathrm{T} 8$ surface gly coproteins. J. Immunol. 131 (1983) 186

Modrow, S., $H$. Wolf: Characterization of two related Epstein-Barr virus-encoded membrane proteins that are differentially expressed in Burkitt lymphoma and in vitro-transformed cell lines. Proc. Natl. Acad. Sci. USA $83(1986) 5703$

Modrow, S., W. Jilg, I. Meerwarth, H. Mairhofer, M. Haus, H. Wolf: Differential expression of EB-viral and cellular surface markers on Burkitt lymphoma and lymphoblastoid cells. In: EBV and its diseases. Humana Press, New Jersey (1987) 407

Motz, M., G. Deby, H. Wolf: Truncated versions of the two major Epstein-Barr viral glycoproteins (gp 250/350) are secreted by recombinant Chinese hamster ovary cells. Gene 58 (1987) 149

Motz, M., J. Fan, R. Seibl, R. Jilg, H. Wolf: Expression of the EpsteinBarr virus $138-\mathrm{kDa}$ early protein in Escherichia coli for the use as antigen in diagnostic tests. Gene 42 (1986) 303

Motz, M., J. Fan, R. Seibl, W. Jilg, H. Wolf: Expression of the EpsteinBarr virus major membrane proteins in Chinese hamster ovary cells. Gene 44 (1986) 353

Purtilo, D. T.: Pathogenesis of an $\mathrm{x}$-linked recessive lymphoproliferative syndrome. Lancet 23 (1976) 882

Raab-Traub, N., K. Flynn: The structure of the termini of the EpsteinBarr Virus as a marker of clonal cellular proliferation. Cell 47 (1986) 883

Rocchi, G., A. de Felici, G. Ragona, A. Heinz: Quantitative evaluation of Epstein-Barr virus-infected mononuclear peripheral blood leukocytes in infectious mononucleosis. N. Engl. J. Med. 296 (1977) 132

Sauerbrei, A., M. Sprößig, P. Wutzler, I. Färber, H. Schweizer, R. Swoboda, J. Wilke: Antikörpertiterverlauf gegen Epstein-Barr-Virus-spezifische Antigene bei infektiöser Mononukleose mit Tonsillektomie in der akuten Krankheitsphase. Laryng. Rhinol. Otol. 62 (1983) 170

Schubach, W. H., R. Hackmann, P. E. Neiman, G. Miller, E. D. Thomas A monoclonal immunoblastic sarcoma in donor cells bearing Epstein-Barr virus genomes following allogenic marrow grafting for acute lymphoblastic leukemia. Blood 60 (1982) 180

Simons, M. J., S. H. Chan, J. H. C. Ho, J. Chan, N. E. Day, G. de Thé: A Singapore 2-associated LD antigen in Chinese patients with Nasopharyngeal carcinoma, In: Histocompatibility Testing. Munksgaard, Copenhagen (1975) 809

Simons, M. J., S. H. Chan, G. B. Wee, K. Shanmugaratnam, E. H. Goh, J. H. C. Ho, J. C. Chau, S. Dharmalingam, U. Prasad, H. Betuel, N. E. Day, G. de Thé: Nasopharyngeal carcinoma and histocompatibility antigens. In: Nasopharyngeal carcinoma. Etiology and Control. De Thé and Ito (eds.) IARC Scientific Publications No. 20, Lyon (1978) 271

Straus, S. E., G. Tosato, G. Armstrong, T. Lawley, O. T. Preble, W. Henle, R. Davey, G. Pearson, J. Epstein, I. Brus, R. M. Blaese: Persisting illness and fatigue in adults with evidence of Epstein-Barr virus infection. In: Ann. of Int. Med., Vol. 102, Nr. 1 (1985) 7

Svedmyr, E., M. Jondal: Cytotoxic effector cells specific for B cell lines transformed by Epstein-Barr virus are present in patients with infectious mononucleosis. Proc. Natl. Acad. Sci. USA 72 (1975) 1622

Taub, R., I. Kirsch, C. Morton et al.: Translocation of the c-myc gene into the immunoglobulin heavy chain locus in human Burkitt's lymphoma and murin plasma-cytoma cells. Proc. Natl. Acad. Sci. USA 79 (1982) 7837

Tobi, M., Z. Ravid, V. Feldman-Weiss, E. Ben-Chetrit, A. Morag, I. Chowers, Y. Michaeli, M. Shalit, H. Krobler: Prolonged atypical illness associated with serological evidence of persistent Epstein-Barr virus infection. In: the Lancet (1982) 61

Tosato, G., I. Magrath, I. Koski, N. Dooley, M. Blaese: Activation of suppressor T-cells during Epstein-Barr virus-induced infectious mononucleosis. N. Engl. J. Med. 301 (1979) 1133 
Wilmes, E., H. Wolf, F. Deinhardt, H. H. Naumann; EBV serology in NPC and related malignancies. In: Nasopharyngeal Carcinoma. Cancer Campaign Vol, 5 Grundmann et al. (eds.), Gustav Fischer Verlag, Stuttgart (1983) 145

Wilmes, E., H. Wolf, M. Haus: Tonsillenkarzinome und Epstein-Barr-Virus. Laryng. Rhinol. Otol, 62 (1983) 586

Wilmes, E., H. Wolf: Der Nachweis von EBV-Genomen in der Ohrspeicheldrüse. Laryng. Rhinol. 60 (1981)

Wolf, H., G. J. Bayliss, E. Wilmes: Biological properties of Epstein-BarrVirus. In: Cancer Campaign. Vol. 5. Nasopharyngeal carcinoma. Grundmann et al. (eds.), Fischer Verlag, Stuttgart (1981) 101

Wolf, H., H. zur Hausen, G. Klein, V. Becker, G. Henle, W. Henle: Attempts to detect virus-specific DNA sequenced in human tumours. III. Epstein-Barr viral DNA in non-lymphoid nasopharygeal carcinoma cells. Med. Microbiol. Immunol, 161 (1975) 15

Wolf, $H ., H$. zur Hausen, V. Becker: EB-viral genomes in epithelial nasopharyngeal carcinoma cells. Nature New Biol. 244 (1973) 245

Wolf, H., M. Haus, E. Wilmes: Brief Communication: On the viral etiology of carcinomas of Waldeyer's ring. In: Nasopharyngeal carcinoma: Current Concepts. Prasad et al. (eds.), University Malaya Press (1983) 277

Wolf, H., M. Haus, E. Wilmes: Persistence of Epstein-Barr virus in the parotid gland. J. Virol. 51 (1984) 795

Wolf, H., M. Motz, R. Kühbeck, W. Jilg, J. Fan, G. H. Pi, Y. Zeng: Development of a set of EBV-specific antigens with recombinant gene technology for diagnosis of EBV-related malignant or nonmalignant diseases. In: EBV and its diseases. Humana Press, New Jersey (1987) 179

Yao, Q. Y., A. B. Rickingson, M. A. Epstein: A re-examination of the Epstein-Barr virus carrier state in healthy seropositive individuals. Int. J. Cancer 35 (1985 a) 35

Zeng, Y., J. M. Zhong, Y. K. Mo, X. C. Miao: Epstein-Barr virus early antigen induction in Raji cells by Chinese medicinal herbs. Intervirology 19 (1983) 201

Ziegler, J. L., R. C. Miner, E. Rosenbaum, E. T. Lennette, E. Schillitoe, C. Casavant, W. L. Drew, L. Mintz, J. Gershow, J. Greenspan, J. Beckstead, K. Yamamoto: Outbreak of Burkitt's-like lymphoma in homosexual men. Lancet, Sept. 18 (1982) 631
Prof. Dr. E. Wilmes

Klinik und Poliklinik

für Hals-, Nasen- und Ohrenkranke

Ludwig-Maximilians-Universität München

Klinikum Großhadern

Marchioninistraße 15

D-8000 München 70 